Finance Stochast. 1, 69-89 (1997)

$\overline{\text { Finance and }}$

Stochastics

(c) Springer-Verlag 1997

\title{
Irreversible investment and industry equilibrium ${ }^{\star}$
}

\author{
Fridrik M. Baldursson ${ }^{1}$, Ioannis Karatzas ${ }^{2}$ \\ ${ }^{1}$ National Economic Institute and the Institute of Economic Studies, University of Iceland, \\ Kalkofnsvegur 1, IS-150 Reykjavik, Iceland (e-mail: fridrikm@centbk.is) \\ 2 Departments of Mathematics and Statistics, Columbia University, New York, N.Y. 10027, USA \\ (e-mail: ik@shire.math.columbia.edu)
}

\begin{abstract}
We establish the equivalence of competitive industry equilibrium with a central planner's decision problem under uncertainty, when investment is irreversible. The existence of industry equilibrium is derived, and it is shown that myopic behavior on the part of small agents is harmless, in the sense that it leads to the same decisions as full rational expectations do. Our model is set in continuous time and allows for very general forms of randomness. The methods are based on the probabilistic approach to singular stochastic control theory and its connections with optimal stopping problems.
\end{abstract}

Key words: Irreversible investment under uncertainty, industry equilibrium, optimality of myopic decisions, singular stochastic control, optimal stopping

JEL classification: E22, D92, G31

Mathematics Subject Classification (1991): 93E20, 60G40, 60G44, 90A16

\section{Introduction}

The purpose of this paper is to extend, and to provide a solid theoretical foundation for, an important part of the theory of irreversible investment. Our methods are based on the probabilistic approach to singular stochastic control theory and its connections with optimal stopping problems.

* The authors gratefully acknowledge partial funding by the Icelandic Science Council, the Nordic Energy Research Programme, and the Fulbright Foundation (to Fridrik M. Baldursson); and by the National Science Foundation under Grant DMS-93-19816 and the U.S. Army Research Office under Grant DAAH 04-95-0528 (to Ioannis Karatzas).

Manuscript received: April 1996; final version received: September 1996 
The theory of irreversible investment under uncertainty has been developed extensively in recent years. The approach has ranged from models of a single investor who faces an exogenous price process, to models of partial equilibrium where price is endogenous. Important contributions to the theory include Arrow (1968) who studied the deterministic case (Dixit 1989a,b; Pindyck 1988, 1991; Leahy 1993; Bertola 1989; Kobila 1991, 1993a, b; Bertola and Caballero 1994). The various approaches and applications are excellently reviewed and extended in a recent book by Dixit and Pindyck (1994).

In this paper we focus on a partial equilibrium model of a competitive industry. Such models have been studied inter alia by Leahy (1993). In Leahy's model the industry is composed of a continuum of infinitesimally small firms which incur irretrievable costs as they enter or exit. The market-clearing output price is determined by an inverse demand curve which is driven by a diffusion process. It is argued that each firm can be myopic as regards future investment in the industry - i.e., can assume that there will be no such investment at all and yet its decisions will be optimal. A similar result was established by Arrow (1968) in a deterministic setting. Leahy's argument did not rely on an "invisible hand" result - i.e. the equivalence of the optimal solution to a social planning problem and the outcome of a decentralized competitive equilibrium - which has been a standard device for characterizing competitive industry equilibria since Lucas and Prescott (1971).

The myopic investor is a strange economic construct in that he models the economy at large correctly, but entertains irrational beliefs about his own sector. Yet, the myopia result is very important since it essentially exogenizes the price process: The price triggers for investment, which are calculated by the myopic investor who takes aggregate supply and demand as exogenous, are still valid for fully rational investors who predict future evolution of supply correctly (see Baldursson (1995) for examples of calculations of competitive and oligopolistic equilibria which exploit this device).

From the viewpoint of applied probability, the investment problem of the small investor is a typical optimal stopping problem. Such problems and their connections to singular stochastic control problems have been studied by analytic methods, at least since Bather and Chernoff (1966) and Beneš et al. (1980). More recent contributions are Karatzas (1983) and Chow et al. (1985). In a series of papers (Karatzas and Shreve 1984, 1985, 1986; Karatzas 1985; Baldursson 1987; El Karoui and Karatzas 1988, 1991) various facets of these problems were studied with probabilistic methods. Although the theory in these papers cannot be brought to bear directly in the setting of models of irreversible investment, the methods can be, and it is one of the main contributions of this paper to do so.

The basic structure of our argument is as follows: First, in Sect. 2, we introduce the small investor's problem and establish some basic identities. Then, in Sect. 3, we use the theory of optimal stopping in continuous time to establish the existence of a solution to the myopic investor's problem.

In Sect. 4 we show that there is a duality between a myopic investor's problem and an appropriate "social planner's" problem whose payoff/cost structure is 
obtained by integrating the payoff/cost functions of the small investor's problem. An explicit characterization of the socially optimal investment process is given in terms of the optimal myopic investment strategy.

In Sect. 5 we show that when the small investor expects the socially optimal investment process to prevail, the myopic investment strategy is also optimal for him. The optimal time of entry happens to coincide with an increase in the social optimum. Furthermore, there are no pure profits to be made under the social optimum, so the small investor is indifferent between entering the industry or staying out. Therefore, the social optimum is also a rational expectations equilibrium.

An extension to the case where capital stock is subject to depreciation or productivity shocks is provided in Sect. 6 . Section 7 concludes.

The theory is developed with full mathematical rigor in continuous time under minimal regularity conditions on the payoff/cost structure. There is no assumption of a Markovian "demand shock", which is the usual assumption in the literature on irreversible investment. The "demand shock" may be infinite-dimensional and depend in an arbitrary way on the past; it is not allowed to anticipate.

\section{The small investor}

Consider a small investor who has the option to invest in an industry. The industry produces a perishable good under decreasing returns to scale with capital as the only production factor. We assume that the investor takes prices as given and maximizes expected profits, given a forecast for the future evolution of prices, or equivalently given an inverse demand function and a forecast of aggregate ${ }^{1}$ demand and industry supply.

To be more specific, let $0<T<\infty$ be a fixed time horizon, $(\Omega, \Im, P)$ a complete probability space, and $\left\{\Im_{t} ; 0 \leq t \leq T\right\}$ a family of $\sigma$-algebras which is increasing in $t, \mathfrak{I}_{t} \subseteq \mathfrak{I}_{s} \subseteq \mathfrak{I}$ for $t \leq s$, right-continuous, and completed by the null sets of $P$. The $\sigma$-algebra $\Im_{t}$ represents the information available to agents at time $t$.

The industry faces an inverse demand curve which is given by the random field, $p(\omega, t, q): \Omega \times[0, T] \times[0, \infty) \rightarrow[0, \infty)$, where $t$ is the time variable, and the random element $\omega$ stands for the state of nature (for example, the path of an aggregate "demand shock"), and $q$ is the demand corresponding to price $p$. Note that the Markovian "demand shock", which is usually assumed to be the source of randomness in models of irreversible investment, is here replaced by the random element $\omega$, which may be infinite-dimensional and may depend on the entire history of events up to time $t$.

Assume that the installed capital stock must be fully utilized at each moment, and select units in such a way that one unit of capital produces one unit of output.

1 Quantities pertaining to the economy at large are referred to as "aggregate", as in "aggregate demand shock". Those pertaining to the industry are tagged as such, for example "industry capital stock". 
Then $p(\omega, t, y)$ is the market-clearing price when $y$ units of capital are installed. We impose the following technical assumptions regarding the inverse demand curve:

$p(\omega, t, y)$ is continuous in $(t, y)$ a.s., progressively measurable

with respect to $\left\{\mathfrak{I}_{t}\right\}$, and decreasing in $y, \forall t \in[0, T]$ a.s. $^{2}$

Furthermore, $E\left[\int_{0}^{T}|p(t, y)| d t\right]<\infty, \quad \forall \quad 0 \leq y<\infty$.

We shall assume there is a continuum of infinitesimally small investors. Each has the option to invest in the industry at some moment $t \in[0, T)$. The options have identical terms: the variable production costs of investors are the same at each instant. ${ }^{3}$ They may, however, depend on the installed capital stock and be subject to random shocks. Denote the per unit variable costs, when $y$ units of capital have been installed, by $c(\omega, t, y): \Omega \times[0, T] \times[0, \infty) \rightarrow[0, \infty)$. We impose the following technical assumption regarding the variable cost curve:

$c(\omega, t, y)$ is continuous in $(t, y)$ a.s., progressively measurable

with respect to $\left\{\Im_{t}\right\}$, and increasing in $y, \forall t \in[0, T]$ a.s.

Furthermore, $E\left[\int_{0}^{T}|c(t, y)| d t\right]<\infty, \quad \forall \quad 0 \leq y<\infty$.

Clearly, the flow of operating surplus $\pi \triangleq p-c$ is decreasing in $y$ and inherits the continuity, measurability and integrability properties of $p$ and $c$.

The cost of exercising the investment option at time $t$ is $\gamma(\omega, t): \Omega \times[0, T] \rightarrow$ $[0, \infty)$. We assume:

$\gamma(\omega, t)$ is non-negative, continuous in $t, P$-a.s. and adapted to $\left\{\mathfrak{I}_{t}\right\}$

(i.e. $\gamma(t)$ is measurable w.r.t. $\left\{\mathfrak{I}_{t}\right\}$ for each $\left.t \in[0, T]\right)$.

Furthermore, $E\left[\sup _{0 \leq t \leq T} \gamma(t)\right]<\infty$.

Finally, $g(\omega, y): \Omega \times[0, \infty) \rightarrow[0, \infty)$ denotes the scrap value of the investment at the time $\mathrm{T}$; we assume that

$$
\begin{aligned}
& g(\omega, y) \text { is continuous and decreasing in } y \text { a.s. and } \\
& \mathfrak{I}_{T} \otimes \text { Borel }([0, \infty)) \text {-measurable; } E[|g(y)|]<\infty, \forall \quad 0 \leq y<\infty \text {. }
\end{aligned}
$$

The conditions (2.1)-(2.4) are assumed to hold throughout the paper.

${ }^{2}$ Progressive measurability of $p$ is here the property that the mapping $(\omega, s, y) \mapsto p(\omega, s, y)$ : $\Omega \times[0, t] \times[0, \infty) \rightarrow[0, \infty)$ be measurable w.r.t. the product $\sigma$-algebra $\Im_{t} \otimes \mathscr{B}_{\text {orel }}([0, t]) \otimes$ Borel $([0, \infty))$, for every $t \in[0, \infty)$.

${ }^{3}$ It is easy to modify the model to allow for different variable costs, but this involves some extra notation which would obscure our basic argument. 
The small investor's problem is to choose an investment strategy, or entry time, $\tau$, to maximize his profits. Such a strategy is simply a mapping $\tau: \Omega \rightarrow$ $[0, T]$ which is such that as time progresses the decision to go ahead with or to delay investment, say at time $t \in[0, T)$, may be made based on information collected up to time $t$, but foresight regarding future random shocks is ruled out: $\{\tau>t\} \in \Im_{t}$. Such a mapping is called a stopping time in the terminology of stochastic processes. In our context, $\tau=T$ means that investment has not been made during $[0, T)$. Denote the collection of stopping times with values in $[0, T]$ by $\mathscr{O}_{0, T}$.

Let $\mathscr{C}$ denote the class of all $\left\{\mathfrak{I}_{t}\right\}$-adapted stochastic processes $\xi$, with nondecreasing, left-continuous sample paths and satisfying $\xi(0)=0$ a.s.

Suppose that a small investor expects cumulative industry investment, beyond the initial capital stock $Y_{0}=y \geq 0$, to follow the process $\xi \in \mathscr{A}$. Assume that capital does not depreciate and that there are no productivity changes, so that installed capital (=output) is simply given by $Y_{t}=y+\xi_{t}$. We shall indicate in Sect. 6 below, how depreciation and productivity changes affect the basic results derived for the present simpler case.

Under the above asumptions, the small investor's expected payoff by using the entry rule $\tau \in \mathscr{O}_{0, T}$ is

$$
\nu(y ; \tau \mid \xi) \triangleq E\left[\int_{t}^{T} \pi\left(t, Y_{t}\right) d t+\left(g\left(Y_{T}\right)-\gamma(\tau)\right) 1_{\{\tau<T\}}\right], \quad y>0,
$$

where we omit the argument corresponding to the random element. The value of the option to enter is

$$
\nu^{*}(y \mid \xi) \triangleq \sup _{\tau \in \mathscr{\mathscr { O }}, T} \nu(y ; \tau \mid \xi) .
$$

We call myopic an investor who assumes that there will be no further investment in the industry, i.e. that $\xi \equiv 0$ (and thus $Y \equiv y$ ). The corresponding payoff

$$
m(y ; \tau) \triangleq \nu(y ; \tau \mid 0)=E\left[\int_{\tau}^{T} \pi(t, y) d t+(g(y)-\gamma(\tau)) 1_{\{\tau<T\}}\right]
$$

is well-defined and finite for every $\tau \in \mathscr{O}_{0, T}$, and we denote the (apparent) option value of the investment opportunity under myopia by

$$
m^{*}(y) \triangleq \sup _{\tau \in \mathscr{O}_{0, T}} m(y ; \tau) \equiv v^{*}(y \mid 0) .
$$

Note that the correct probability measure is used - the myopia is limited to future investment in the industry the investor may want to invest in.

It is convenient to define the expected opportunity cost of using a particular investment strategy:

$$
\rho(y ; \tau \mid \xi)=E\left[\int_{0}^{\tau} \pi\left(t, Y_{t}\right) d t+\gamma(\tau) 1_{\{\tau<T\}}+g\left(Y_{T}\right) 1_{\{\tau=T\}}\right], \quad y<0 .
$$

Let 


$$
\rho^{*}(y \mid \xi) \triangleq \inf _{\tau \in \mathscr{K}, T} \rho(y ; \tau \mid \xi),
$$

and also denote the corresponding entities associated with the myopic investor by

$$
\begin{aligned}
& r(y ; \tau) \triangleq \rho(y ; \tau \mid 0)=E\left[\int_{0}^{\tau} \pi(t, y) d t+\gamma(\tau) 1_{\{\tau<T\}}+g(y) 1_{\{\tau=T\}}\right] \\
& r^{*}(y) \triangleq \inf _{\tau \in \mathscr{V}, T} r(y ; \tau) \equiv \rho^{*}(y \mid 0) .
\end{aligned}
$$

Note the identities

$$
\begin{aligned}
& \rho(y ; T \mid \xi) \equiv \nu(y ; 0 \mid \xi)+E[\gamma(0)] \equiv \rho(y ; \tau \mid \xi)+\nu(y ; \tau \mid \xi), \\
& r(y ; T) \equiv m(y ; T)+E[\gamma(0)] \equiv r(y ; \tau)+m(y ; \tau),
\end{aligned}
$$

which are valid for any $\tau \in \mathscr{O}_{0, T}$, and hence also

$$
\begin{aligned}
& \rho(y ; T \mid \xi) \equiv \nu(y ; 0 \mid \xi)+E[\gamma(0)] \equiv \rho^{*}(y ; \mid \xi)+\nu^{*}(y ; \mid \xi), \\
& r(y ; T) \equiv m(y ; T)+E[\gamma(0)] \equiv r^{*}(y ; \tau)+m^{*}(y) .
\end{aligned}
$$

Clearly, maximization of $\nu$ and $m$ is equivalent to minimization of $\rho$ and $r$, respectively. Note that, since for any $\tau \in \mathscr{S}_{0, T}$ and $\xi \in \mathscr{\ell}$ we have

$$
\begin{aligned}
& \nu(y ; \tau \mid \xi) \leq m(y ; \tau)<\infty \\
& \rho(y ; \tau \mid \xi) \leq r(y ; \tau)<\infty
\end{aligned}
$$

the investment payoff and risk, $\nu(y ; \tau \mid \xi)$ and $\rho(v ; \tau \mid \xi)$, respectively, are always well-defined, for an arbitrary $\xi \in \mathscr{\ell}$, but their value may be minus infinity.

\section{The myopic solution}

In this section we shall show that there exists a solution to the myopic investor's problem, and we shall characterize this solution, under minimal regularity conditions. We rely on the theory of optimal stopping in continuous time (Shiryayev 1978; El Karoui 1981; Karatzas 1993).

For each fixed $y>0$ let

$$
R^{y}(t) \triangleq \int_{0}^{t} \pi(s, y) d s+\gamma(t) 1_{\{t<T\}}+g(y) 1_{\{t=T\}}, \quad t \in[0, T]
$$

and note that $r(y ; \tau)=E\left[R^{y}(\tau)\right], \tau \in \mathscr{C}_{0, T}$. Recall that $\pi(\omega, t, y)$ is a.s. continuous in $(t, y)$, so that the random field $\pi_{L}(y) \triangleq \min _{0 \leq t \leq T} \pi(t, y)$ is well-defined. We shall make the regularity assumption

$$
E\left[\pi_{L}(y)\right]>-\infty, \quad y>0
$$

We also assume 


$$
\gamma(T) \geq g(0), \quad \text { a.s. }
$$

While (3.2) is a genuine regularity condition which can probably be relaxed with further effort, a violation of (3.3) would imply that no optimal investment time for the myopic investor need exist. This possibility arises when $\gamma$ is decreasing and $\pi$ is non-negative or small in absolute value, so at each time strictly before $T$ profits can be further increased by waiting a little longer (consider the case $\pi \equiv 0, \gamma(t)=T /(t+T), g \equiv 1)$.

Recall from (2.4) that $g(\omega, y)$ is decreasing in $y$, so (3.3) implies $\gamma(T) \geq g(y)$ a.s. for each $y>0$.

Further, note that $R^{y}(\omega, t)$ is continuous in $t$ on $[0, T)$, while by (3.3):

$$
R^{y}(T)-\lim _{t \uparrow T} R^{y}(t)=g(y)-\gamma(T) \leq 0 \quad \text { a.s. }
$$

For each $y>0$, let

$$
Z^{y}(t) \triangleq \underset{\tau \in \mathscr{T}, T}{\operatorname{essinf}} \quad E\left[R^{y}(\tau) \mid \Im_{t}\right], \quad 0 \leq t \leq T,
$$

where $\mathscr{T}_{t, T} \triangleq\left\{\tau \in \mathscr{\mathscr { O }}_{0, T} ; \tau \geq t\right.$ a.s. $\}$. Given that entry has not taken place prior to time $t$, and conditioning on information collected up to that instant, $Z^{y}(t)$ is the lowest (conditional) expected cost one can incur in the future.

As shown in El Karoui (1981) and Karatzas (1993) (Theorems 2.7 and 3.3 in Chapter 3), the conditions (3.2)-(3.4) and the continuity of $t \mapsto R^{y}(\gamma, t)$ on $[0, T)$ are sufficient to establish the following proposition, phrased in the context of our particular problem.

Proposition 1: Assume that (2.1)-(2.4) and (3.2)-(3.3) hold. Then for any given $y>0$, there exists a submartingale $Z_{0}^{y}(\cdot)$, with sample paths which are almost surely right-continuous on $[0, T)$ and admit left-hand limits everywhere on $(0, T]$, and is such that

$$
Z_{0}^{y}(t)=Z^{y}(t), \quad \text { a.s. for all } t \in[0, T] .
$$

Furthermore, $Z_{0}^{y}(t) \leq R^{y}(t)$ a.s. for all $t \in[0, T]$, and the stopping time

$$
\sigma(y) \triangleq \inf \left\{t \in[0, T) ; Z_{0}^{y}(t)=R^{y}(t)\right\} \wedge T
$$

is an optimal investment strategy for the myopic investor:

$$
m(y ; \sigma(y))=m^{*}(y) .
$$

The supermartingale $-Z_{0}^{y}$ is usually referred to as the Snell-envelope of $-R^{y}$, after Snell (1952).

It is intuitively clear that the higher the initial capital stock - and, therefore, the lower the initial operating surplus - the longer one should wait to enter. This turns out to be a useful property, so we state and prove it formally. 
Lemma 1: Assume that (2.1)-(2.4) and (3.2)-(3.3) hold. Then the mapping $y \mapsto$ $\sigma(y)$ is increasing a.s.

Proof. Define, for each $t \in[0, T]$ and $y>0$, by analogy with (2.8), (2.8)':

$$
u(t, y) \triangleq \underset{\tau \in \mathscr{T}_{t, T}}{\operatorname{essinf} E}\left[\int_{t}^{\tau} \pi(s, y) d s+\gamma(\tau) 1_{\{\tau<T\}}+g(y) 1_{\{\tau=T\}} \mid \Im_{t}\right],
$$

so that $u(0, y)=r^{*}(y)$. Note that for $t \in[0, T]$ and $y>0$ we have

$$
Z_{0}^{y}(t)-R^{y}(t)=u(t, y)-\gamma(t) 1_{\{t<T\}}-g(y) 1_{\{t=T\}}, \quad \text { a.s. }
$$

Therefore, $u(t, y) \leq \gamma(t)$ a.s., for $t \in[0, T)$, and

$$
\sigma(y)=\inf \{t \in[0, T) ; u(t, y)=\gamma(t)\} \wedge T, \quad \text { a.s. }
$$

Also note that $u(t, y)$ is decreasing in $y$ a.s., since $\pi(t, y)$ and $g(y)$ are. The conclusion of the lemma follows.

\section{The social planning problem}

Let us recall the definition of $\mathscr{C}$, the class of cumulative investment processes the small investors expect to encounter. We take now the point of view that these processes correspond to admissible "industry investment policies" available to a fictitious "social planner". The expected social surplus corresponding to such an industry investment policy is given by

$$
J(y ; \xi) \triangleq E\left[\int_{0}^{T} \Pi\left(t, Y_{t}\right) d t-\int_{[0, T)} \gamma(t) d \xi_{t}+G\left(Y_{T}\right)\right],
$$

where $Y_{t}=y+\xi_{t}$ is the corresponding industry capital stock at time $t$, and where we have set

$$
\begin{aligned}
& U(\omega, t, y) \triangleq \int_{1}^{y} p(\omega, t, x) d x \quad \text { (flow of consumers' surplus) } \\
& C(\omega, t, y) \triangleq \int_{1}^{y} c(\omega, t, x) d x \text { (flow of total variable production costs) } \\
& \Pi \triangleq U-C \quad \text { (flow of social “operating surplus") } \\
& G(\omega, y) \triangleq \int_{1}^{y} g(\omega, x) d x \quad \text { (scrap value of industry capital stock). }
\end{aligned}
$$

Note the concavity of the random fields $U, \Pi$ and $G$ in the spatial variable $y$. Clearly, the expected benefit of not investing at all is given by

$$
I(y) \triangleq J(y ; 0)=E\left[\int_{0}^{T} \Pi(t, y) d t+G(y)\right] .
$$


The purpose of this fictitious "social planner" is to choose the (irreversible industry investment) policy $\xi$, in such a way as to maximize expected social surplus. Thus, he faces a stochastic control problem of the monotone follower type, with value function given by

$$
V(y) \triangleq \sup _{\xi \in \mathscr{C}} J(y ; \xi) .
$$

In order to ensure the validity of the definition $(4.3)^{\prime}$, we shall assume:

$$
\begin{gathered}
\sup _{y>0} \Pi(t, y)^{+} \text {and } \sup _{y \geq 0} G(y)^{+} \text {are integrable on } \Omega \times[0, T] \\
\text { and } \Omega, \text { respectively }
\end{gathered}
$$

In this section we shall study the relation of this "social planner's" optimization problem (4.3)' with the small investor's optimal stopping problem of Sect. 2. The approach will be based on the methodology of El Karoui and Karatzas (1991).

For any given $\xi \in \mathscr{\ell}$, we consider its right-continuous inverse

$$
\tau^{\xi}(x) \triangleq \inf \left\{t \in[0, T) ; \xi_{t}>x\right\} \wedge T, \quad x \geq 0
$$

Note that $\tau^{\xi}(x) \in \mathscr{T}_{0, T}$ for every $x \geq 0$, and that

$$
\tau^{\xi}(x)<t \Leftrightarrow \xi_{t}>x, \quad \text { for } 0 \leq t \leq T .
$$

In particular, (4.6) leads, via a monotone class argument, to the change-ofvariable formula

$$
\int_{0}^{\infty} h\left(\tau^{\xi}(x)\right) 1_{\left\{\tau^{\xi}(x)<T\right\}} d x=\int_{[0, T)} h(t) d \xi_{t} \quad \text { a.s. }
$$

for any Borel-measurable function $h:[0, T] \rightarrow[0, \infty)$. Observe also from (4.6) that we can reproduce $\xi$ as the left-continuous inverse of $\tau^{\xi}(\cdot)$ :

$$
\xi_{t}=\sup \left\{x \in[0, \infty) ; \tau^{\xi}(x)<t\right\} \quad 0 \leq t \leq T .
$$

Lemma 2: Assume that (2.1)-(2.4), (3.2)-(3.3) and (4.4) hold. For any given $y>0$ and $\xi \in \mathscr{C}$, we have

$$
\begin{gathered}
J(y ; \xi)-I(y)=\int_{y}^{\infty} m\left(x ; \tau^{\xi}(x-y)\right) d x, \\
V(y)-I(y) \leq \int_{y}^{\infty} m^{*}(x) d x .
\end{gathered}
$$

Proof: We write $\tau$ for $\tau^{\xi}$ in this proof. From (4.7) we get

$$
\int_{y}^{\infty} \gamma(\tau(x-y)) 1_{\{\tau(x-y)<T\}} d x=\int_{[0, T)} \gamma(t) d \xi_{t} \quad \text { a.s. }
$$

Similarly, from (4.6) and the Fubini theorem we obtain: 


$$
\begin{aligned}
\int_{y}^{\infty} \int_{\tau(x-y)}^{T} \pi(t, x) d t d x & \equiv \int_{y}^{\infty} \int_{0}^{T} \pi(t, x) 1_{\{\tau(x-y)<t\}} d t d x \\
& \equiv \int_{0}^{T} \int_{y}^{\infty} \pi(t, x) 1_{\left\{\xi_{t}>x-y\right\}} d x d t \\
& \equiv \int_{0}^{T}\left(\Pi\left(t, y+\xi_{t}\right)-\Pi(t, y)\right) d t \\
& \equiv \int_{0}^{T}\left(\Pi\left(t, Y_{t}\right)-\Pi(t, y)\right) d t
\end{aligned}
$$

In the same way we obtain,

$$
\int_{y}^{\infty} g(x) 1_{\{\tau(x-y)<T\}} d x=G\left(y+\xi_{T}\right)-G(y)=G\left(Y_{T}\right)-G(y) .
$$

Therefore, by (4.10)-(4.12),

$$
\begin{aligned}
& \int_{y}^{\infty} E {\left[\int_{\tau(x-y)}^{T} \pi(t, x) d t+(g(x)-\gamma(\tau(x-y))) 1_{\{\tau(x-y)<T\}}\right] d x } \\
& \equiv E {\left[\int_{0}^{T} \Pi\left(t, Y_{t}\right) d t-\int_{[0, T)} \gamma(t) d \xi_{t}+G\left(Y_{T}\right)\right] } \\
& \quad-E\left[\int_{0}^{T} \Pi(t, y) d t+G(y)\right] \\
& \equiv J(y ; \xi)-I(y) .
\end{aligned}
$$

Equation (4.8) now follows from (2.5) and the definition of $m(y ; \tau)$. The inequality (4.9) is easily obtained, by first observing that $m(y ; \tau) \leq m^{*}(y)$, and then taking the supremum over $\xi \in \mathscr{A}$.

Now, for any given $y>0$, consider the family of stopping times

$$
\tau_{y}(x) \triangleq \sigma(x+y), \quad x \in[0, \infty)
$$

where $\{\sigma(z) ; z>0\}$ is the family of stopping times defined by (3.7) (see also (3.10)). Let $\xi_{y}^{*}(\cdot) \in \mathscr{\ell}$ be the left-continuous inverse of $\tau_{y}(\cdot)$, defined by analogy with $(4.6)^{\prime}$ as

$$
\begin{aligned}
\xi_{y}^{*}(t) & \triangleq \sup \left\{x \in[0, \infty) ; \tau_{y}(x+)<t\right\} \\
& =\sup \{z \in[y, \infty) ; \sigma(z+)<t\}, \quad 0 \leq t \leq T .
\end{aligned}
$$

It may aid in the intuitive understanding of this last definition to think of the continuum of small investors "lined up" to the right of $y$ indexed by the corresponding initial capital stock $Y_{0}=z \geq y$. At time $t$, the aggregate industrial investment $\xi_{y}^{*}(t)$ is given by the location of the "marginal", myopic investor who is "last" to enter by time $t$, i.e., who is located furthest to the right of those who 


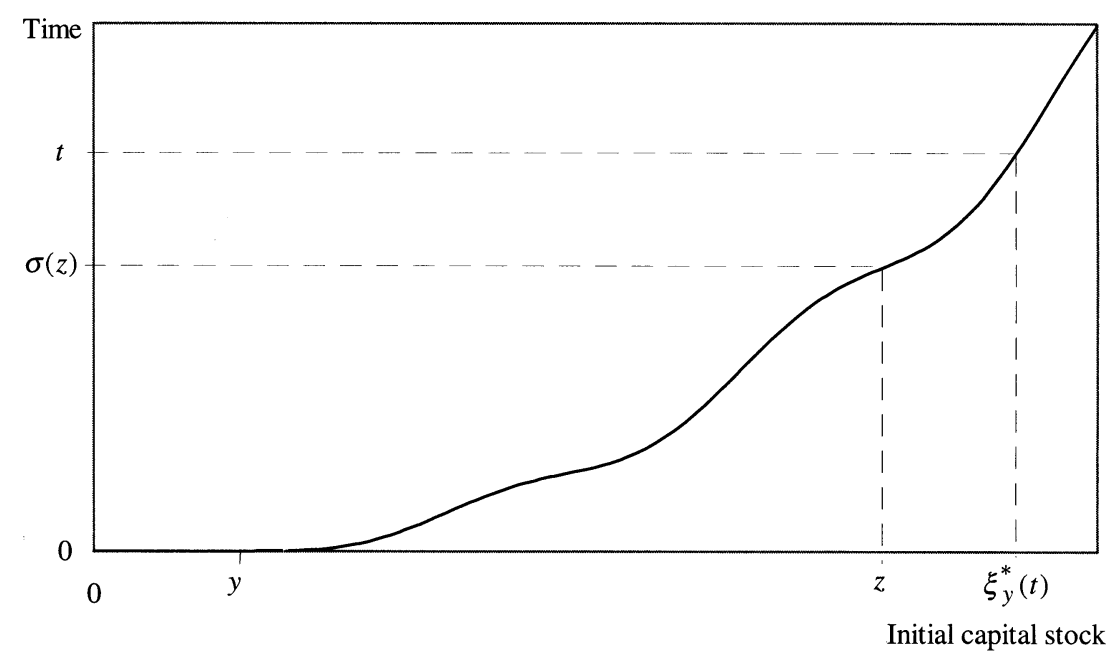

Fig. 1. With initial capital stock $Y_{0}=z$ the corresponding myopic investor would have entered by time $t$. Therefore $z \leq \xi_{y}^{*}(t)$, since $\xi_{y}^{*}(t)$ is the largest such number

would have entered by time $t$, according to their myopic strategies (see Fig. 1). We owe this interpretation to Professor Roy Radner.

We have from $(4.3)^{\prime},(4.8),(3.8)$ and (4.9):

$$
\begin{aligned}
V(y)-I(y) \geq J\left(y ; \xi_{y}^{*}\right)-I(y) & =\int_{y}^{\infty} m\left(x ; \tau_{y}(x-y)\right) d x \\
& =\int_{y}^{\infty} m(x ; \sigma(x)) d x \\
& =\int_{y}^{\infty} m^{*}(x) d x \\
& \geq V(y)-I(y) .
\end{aligned}
$$

We are led to the following result:

Theorem 1: Assume that (2.1)-(2.4), (3.2)-(3.3) and (4.4) hold. Then, for any given $y>0$, there exists an optimal investment process $\xi_{y}^{*}(\cdot)$ for the "social planner's" problem of $(4.3)^{\prime}$, and it is given by (4.15). Furthermore, for all $y>0$,

$$
\begin{gathered}
V(y)=I(y)+\int_{y}^{\infty} m^{*}(x) d x \\
V^{\prime}(y)=r^{*}(y) \\
\sigma(y+)=\inf \left\{t \in[0, T) ; \xi_{y}^{*}(t)>0\right\} \wedge T .
\end{gathered}
$$

Proof: Equation (4.17), and the optimality of $\xi_{y}^{*}(\cdot)$ for the problem of $(4.3)^{\prime}$, follow directly from (4.16). The relation (4.18) comes readily from (4.17), (2.11) and 


$$
I^{\prime}(y)=E\left[\int_{0}^{T} \pi(t, y) d t+g(y)\right]=r(y ; T)
$$

which is easily verified by considering $I(y \pm \varepsilon)-I(y)$ for small $\varepsilon>0$. Equation (4.19) follows from the definition of $\xi_{y}^{*}(\cdot)$ and (4.6).

\section{Equilibrium}

In the preceding section we established and exploited the duality between the myopic investor's problem and the social planning problem, to prove the existence of a socially optimal investment process $\xi_{y}^{*}(\cdot)$. We showed that the social value of a marginal unit of capital equals the optimal expected opportunity cost of a myopic investor (cf. relation (4.18)). We characterized the optimal cumulative investment $\xi_{y}^{*}(t)$ for the "social planner" at time $t$, as the largest value of initial investment $Y_{0}=z \geq y$ that would make a "myopic" small investor decide to enter by time $t$ (cf. (4.15)). We also came very close to showing that a myopic investor at $Y_{0}=y$ should act exactly at the same time as the social planner does (cf. relation (4.19)).

In this section we take a different approach, similar to that of Karatzas and Shreve (1984), to establish that, when a small investor at $Y_{0}=y>0$ forecasts the socially optimal investment process $\xi_{y}^{*}(\cdot)$ to prevail, the optimal time of entry for him is the first time $\xi_{y}^{*}(t)>0$; and that this is, in fact, an optimal entry time for a myopic investor as well. Furthermore, the value of the option to invest in the industry is zero with this forecast (i.e. expecting $\xi_{y}^{*}(\cdot)$ as the investment process), and hence the investor will be indifferent between entering and holding his capital in bonds. Therefore, $\xi_{y}^{*}(\cdot)$ is a rational expectations equilibrium investment process. We summarize these findings in the following theorem.

Theorem 2: Assume that (2.1)-(2.4), (3.2)-(3.3) and (4.4) hold. Then for any given $y>0$ :

(i) We have

$$
V^{\prime}(y)=r^{*}(y)=\rho^{*}\left(y \mid \xi_{y}^{*}\right) .
$$

(ii) The entry time

$$
\tau_{y}^{*} \triangleq \inf \left\{t \in[0, T) ; \xi_{y}^{*}(t)>0\right\} \wedge T
$$

is optimal both for a "myopic investor" (who forecasts that $\xi \equiv 0$ will prevail) and for a "small investor" who forecasts that the "socially optimal" strategy $\xi \equiv \xi_{y}^{*}(\cdot)$ will prevail. In other words, $\rho^{*}(y \mid \xi)=\rho\left(y, \tau_{y}^{*} \mid \xi\right)$ for both $\xi \equiv 0$ and $\xi \equiv \xi_{y}^{*}(\cdot)$.

(iii) If, in addition, there exists an $\varepsilon_{0}>0$ such that $\nu\left(y+\varepsilon_{0} ; T \mid \xi_{y}^{*}\right)>-\infty$, then

$$
\nu^{*}\left(y \mid \xi_{y}^{*}\right)=0
$$


In other words, for a small investor who forecasts that the "socially optimal" strategy $\xi \equiv \xi_{y}^{*}(\cdot)$ will prevail, the value of the option to enter the industry is zero.

Note that $\sigma(y+)$ of Sect. 4 equals $\tau_{y}^{*}$ (recall (4.19) of Theorem 1). We prove Theorem 2 in a series of Lemmata.

Lemma 3: Assume that (2.1)-(2.4), (3.2)-(3.3) and (4.4) hold. Then for any given $y>0$ and $\tau \in \mathscr{C}_{0, T}$, we have

$$
V^{\prime}(y) \leq \rho\left(y ; \tau \mid \xi_{y}^{*}\right) \leq r(y ; \tau) .
$$

Proof: Take arbitrary $y>\varepsilon>0$ and $\tau \in \mathscr{T}_{0, T}$. Let $\xi \equiv \xi_{y}^{*}, Y \equiv y+\xi$, $Y^{-\varepsilon} \equiv y-\varepsilon+\xi$ and define

$$
\eta_{t}=\left\{\begin{array}{ccc}
\xi(t) & ; \quad 0 \leq t \leq \tau \\
\xi(t)+\varepsilon & ; \quad \tau<t \leq T
\end{array}\right\} .
$$

It is easily seen that $\eta \in \mathscr{C}$, and that the corresponding state process $Z=y-\varepsilon+\eta$ follows the path of $Y^{-\varepsilon}$ up to time $\tau$ and then switches to the path of the process $Y$. The performance associated with $(y-\varepsilon, \eta)$ may be written as

$$
\begin{aligned}
& J(y-\varepsilon ; \eta)=E\left[\int_{0}^{T} \Pi\left(t, Z_{t}\right) d t-\int_{[0, T)} \gamma(t) d \eta_{t}+G\left(Z_{T}\right)\right] \\
& =E\left[\int_{0}^{\tau} \Pi\left(t, Y_{t}^{-\varepsilon}\right) d t+\int_{\tau}^{T} \Pi\left(t, Y_{t}\right) d t-\int_{[0, T)} \gamma(t) d \xi_{t}-\varepsilon \gamma(\tau) 1_{\{\tau<T\}}\right. \\
& \left.\quad+G\left(Y_{T}\right) 1_{\{\tau<T\}}+G\left(Y_{T}^{-\varepsilon}\right) 1_{\{\tau=T\}}\right]
\end{aligned}
$$

Comparing the expressions (4.1) and (5.6) we obtain

$$
\begin{aligned}
V(y)-V(y-\varepsilon)=J(y ; \xi)-V(y-\varepsilon) \leq J(y ; \xi)-J(y-\varepsilon ; \eta) \\
=E\left[\int_{0}^{\tau}\left(\Pi\left(t, Y_{t}\right)-\Pi\left(t, Y_{t}^{-\varepsilon}\right)\right) d t+\varepsilon \gamma(\tau) 1_{\{\tau<T\}}\right. \\
\left.\quad \quad \quad+\left(G\left(Y_{T}\right)-G\left(Y_{T}^{-\varepsilon}\right)\right) 1_{\{\tau=T\}}\right] \\
\leq \varepsilon E\left[\int_{0}^{\tau} \pi\left(t, y-\varepsilon+\xi_{t}\right) d t+\gamma(\tau) 1_{\{\tau<T\}}+g\left(y-\varepsilon+\xi_{T}\right) 1_{\{\tau=T\}}\right] \\
=\varepsilon \rho(y-\varepsilon ; \tau \mid \xi) \\
\leq \varepsilon \cdot E\left[\int_{0}^{\tau} \pi(t, y-\varepsilon) d t+\gamma(\tau) 1_{\{\tau<T\}}+g(y-\varepsilon) 1_{\{\tau=T\}}\right] \\
=\varepsilon \cdot r(y-\varepsilon ; \tau)
\end{aligned}
$$

where we have used the concavity of $\Pi(t, \cdot)$ and $G(\cdot)$ (or equivalently the decrease of $\pi(t, \cdot)$ and $g(\cdot))$. Now divide through by $\varepsilon$, let $\varepsilon \downarrow 0$, and use the monotone convergence theorem to get the inequalities of (5.4). The second inequality follows easily from the monotonicity of $\pi(t, \cdot)$ and $g(\cdot)$. 
Lemma 4: Assume that (2.1)-(2.4), (3.2)-(3.3) and (4.4) hold. Then, for any given $y>0$, we have

$$
V^{\prime}(y) \geq r\left(y ; \tau_{y}^{*}\right)=\rho\left(y ; \tau_{y}^{*} \mid \xi_{y}^{*}\right) .
$$

Proof: Let $\xi \equiv \xi_{y}^{*}$. In addition to the stopping time $\tau_{y}^{*}$ of (5.2) we introduce the family of stopping times, defined for all $\varepsilon>0$,

$$
\begin{aligned}
\tau^{\varepsilon} & \triangleq \inf \left\{t \in[0, T) ; Y_{t} \geq y+\varepsilon\right\} \wedge T \\
& =\inf \{t \in[0, T) ; \xi(t) \geq \varepsilon\} \wedge T .
\end{aligned}
$$

Observe that $\tau^{\varepsilon} \downarrow \tau^{*}$ almost surely, as $\varepsilon \downarrow 0$. For every fixed $\varepsilon>0$ we create the capital stock process $Z \equiv y+\varepsilon+\eta$, where $\eta \in \mathscr{C}$ is the investment process

$$
\eta(t) \triangleq\left\{\begin{array}{ccc}
0 & ; \quad 0 \leq t \leq \tau^{\varepsilon} \\
\xi(t)-\varepsilon & ; \quad \tau^{\varepsilon}<t \leq T
\end{array}\right\} .
$$

The reader will observe that $Z$ starts at $y+\varepsilon$, stays there until time $\tau_{\varepsilon}$, and then switches to the process $Y$, which by then has exceeded $y+\varepsilon$ :

$$
Z_{t}=\left\{\begin{array}{ccc}
y+\varepsilon & ; \quad 0 \leq t \leq \tau^{\varepsilon} \\
Y_{t} & ; \quad \tau^{\varepsilon}<t \leq T
\end{array}\right\} .
$$

The performance (expected surplus) corresponding to this policy is given by

$$
\begin{aligned}
J(y+\varepsilon ; \eta) & =E\left[\int_{0}^{T} \Pi\left(t, Z_{t}\right) d t-\int_{[0, T)} \gamma(t) d \eta(t)+G\left(Z_{T}\right)\right] \\
& =E\left[\int_{0}^{\tau^{\varepsilon}} \Pi(t, y+\varepsilon) d t+\int_{\tau^{\varepsilon}}^{T} \Pi\left(t, Y_{t}\right) d t-\gamma\left(\tau^{\varepsilon}\right) \eta\left(\tau^{\varepsilon}+\right) 1_{\left\{\tau^{\varepsilon}<T\right\}}\right. \\
& \left.-\int_{\left(\tau^{\varepsilon}, T\right)} \gamma(t) d \xi(t)+G(y+\varepsilon) 1_{\left\{\tau^{\varepsilon}=T\right\}}+G\left(Y_{T}\right) 1_{\left\{\tau^{\varepsilon}<T\right\}}\right] .
\end{aligned}
$$

Since $J(y ; \xi)=Y(y)$ we have also

$$
\begin{aligned}
V(y) & =E\left[\int_{0}^{\tau^{\varepsilon}} \Pi\left(t, Y_{t}\right) d t+\int_{\tau^{\varepsilon}}^{T} \Pi\left(t, Y_{t}\right) d t\right. \\
& -\int_{\left[0, \tau^{\varepsilon}\right)} \gamma(t) d \xi(t)-\gamma\left(\tau^{\varepsilon}\right)\left(\xi\left(\tau^{\varepsilon}+\right)-\xi\left(\tau^{\varepsilon}\right)\right) 1_{\left(\tau^{\varepsilon}<T\right)}-\int_{\left(\tau^{\varepsilon}, T\right)} \gamma(t) d \xi(t) \\
& \left.+G\left(Y_{T}\right) 1_{\left\{\tau^{\varepsilon}=T\right\}}+G\left(Y_{T}\right) 1_{\left\{\tau^{\varepsilon}<T\right\}}\right],
\end{aligned}
$$

and comparing (5.12) with (5.13) we obtain

$$
\begin{aligned}
& V(y+\varepsilon)-V(y) \geq J(y+\varepsilon ; \eta)-V(y) \\
& \quad=E\left[\int_{0}^{\tau^{\varepsilon}}(\Pi(t, y+\varepsilon)-\Pi(t, y+\xi(t))) d t+\int_{\left[0, \tau^{\varepsilon}\right)} \gamma(t) d \xi(t)\right. \\
& \left.\quad+\gamma\left(\tau^{\varepsilon}\right)\left\{\varepsilon-\xi\left(\tau^{\varepsilon}\right)\right\} 1_{\left\{\tau^{\varepsilon}<T\right\}}+(G(y+\varepsilon)-G(y+\xi(T))) 1_{\left\{\tau^{\varepsilon}=T\right\}}\right] .
\end{aligned}
$$


Now the concavity of $\Pi(t, \cdot)$ yields

$$
\Pi(t, y+\varepsilon)-\Pi(t, y+\xi(t)) \geq(\varepsilon-\xi(t)) \cdot \pi(t, y+\varepsilon)
$$

almost surely, and a similar expression obtains for $G$. In light of (5.15), the inequality (5.14) becomes

$$
\begin{aligned}
& V(y+\varepsilon)-V(y) \geq J(y+\varepsilon ; \eta)-V(y) \\
& =E\left[\int_{0}^{\tau^{\varepsilon}}(\varepsilon-\xi(t)) \cdot \pi(t, y+\varepsilon) d t+\int_{\left[0, \tau^{\varepsilon}\right)} \gamma(t) d \xi(t)\right. \\
& \left.\quad+\gamma\left(\tau^{\varepsilon}\right)\left(\varepsilon-\xi\left(\tau^{\varepsilon}\right)\right) 1_{\left\{\tau^{\varepsilon}<T\right\}}+(\varepsilon-\xi(T)) \cdot g(y+\varepsilon) 1_{\left\{\tau^{\varepsilon}=T\right\}}\right]
\end{aligned}
$$

Interchanging a few terms, we write (5.16) as

$$
\frac{V(y+\varepsilon)-V(y)}{\varepsilon} \geq r\left(y ; \tau^{*}\right)+\sum_{j=1}^{6} I_{j}(\varepsilon)
$$

where

$$
\begin{aligned}
& I_{1}(\varepsilon)=E\left[\int_{0}^{\tau^{*}}\{\pi(t, y+\varepsilon)-\pi(t, y)\} d t\right], \\
& I_{2}(\varepsilon)=E\left[\int_{\tau^{*}}^{\tau^{\varepsilon}} \pi(t, y+\varepsilon)\{1-(\xi(t) / \varepsilon)\} d t\right], \\
& I_{3}(\varepsilon)=E\left[\{g(y+\varepsilon)-g(y)\} 1_{\left\{\tau^{*}=T\right\}}\right], \\
& I_{4}(\varepsilon)=E\left[g(y+\varepsilon)\{1-(\xi(T) / \varepsilon)\} 1_{\left\{\tau^{*}<\tau^{\varepsilon}=T\right\}}\right], \\
& I_{5}(\varepsilon)=E\left[\gamma\left(\tau^{\varepsilon}\right) 1_{\left\{\tau^{\varepsilon}<T\right\}}-\gamma\left(\tau^{*}\right) 1_{\left\{\tau^{*}<T\right\}}\right], \\
& I_{6}(\varepsilon)=\frac{1}{\varepsilon} E\left[\int_{\left(\tau^{*}, \tau^{\varepsilon}\right)} \gamma(t) d \xi(t)-\gamma\left(\tau_{\varepsilon}\right)\left(\xi\left(\tau^{\varepsilon}\right)-\xi\left(\tau^{*}\right)\right)\right] .
\end{aligned}
$$

It is not hard to verify that $\lim _{\varepsilon \downarrow 0} I_{j}(\varepsilon)=0$ for each $j=1, \ldots, 6$; one uses the continuity properties of the functions $\pi(t, y), g(y)$ and $\gamma(t)$, the regularity conditions (2.1)-(2.4), the fact that $\left\{\tau^{*}<\tau^{\varepsilon}=T\right\} \downarrow \varnothing$ as $\varepsilon \downarrow 0$, the definition of $\tau^{\varepsilon}$ which entails $0 \leq \xi(t) \leq \varepsilon$ a.s. on $\left\{t<\tau^{\varepsilon}\right\}$, and the dominated and monotone convergence theorems. Now we let $\varepsilon \downarrow 0$ in (5.17), to obtain the inequality of (5.8); the equality in (5.8) is a consequence of the definition (5.2) of $\tau_{y}^{*}$.

Lemma 5: Assume that (2.1)-(2.4), (3.2)-(3.3) and (4.4) hold, and that for every $y>0$, there exists $\varepsilon_{0}=\varepsilon_{0}(y)>0$ such that

$$
\nu\left(y+\varepsilon_{0} ; T \mid \xi_{y}^{*}\right)>-\infty .
$$

Then

$$
V^{\prime}(y) \geq \rho\left(y ; T \mid \xi_{y}^{*}\right), \text { for all } y>0
$$


Proof: Let $y>0$, pick $\varepsilon_{0}=\varepsilon_{0}(y)>0$ such that (5.18) holds and let $\varepsilon \in\left(0, \varepsilon_{0}\right)$. By $(4.3)^{\prime}$, the optimality of $\xi \equiv \xi_{y}^{*}$ for the "social planner's" problem, and the concavity of $\Pi(t, \cdot)$ and $G(\cdot)$, we have:

$$
\begin{aligned}
& (V(y+\varepsilon)-V(y)) / \varepsilon \geq(J(y+\varepsilon ; \xi)-J(y ; \xi)) / \varepsilon \\
& \geq E\left[\int_{0}^{T} \pi(t, y+\varepsilon+\xi(t)) d t+g(y+\varepsilon+\xi(T))\right] .
\end{aligned}
$$

Observe that by the continuity and monotonicity of $\pi(t, \cdot)$ and $g(\cdot)$ we have that, as $\varepsilon \downarrow 0$,

$$
\begin{gathered}
\pi\left(t, y+\varepsilon_{0}+\xi(t)\right) \leq \pi(t, y+\varepsilon+\xi(t)) \uparrow \pi(t, y+\xi(t)) \quad \text { a.s. } \forall \quad t \in[0, T] \\
g\left(y+\varepsilon_{0}+\xi(T)\right) \leq g(y+\varepsilon+\xi(T)) \uparrow g(y+\xi(T)) \quad \text { a.s. }
\end{gathered}
$$

Now we let $\varepsilon \downarrow 0$ in (5.20) and use (5.21) and the monotone convergence theorem to obtain the inequality (5.19).

Proof of Theorem 2: By Lemmata 3 and 4, we have

$$
r\left(y ; \tau_{y}^{*}\right)=\rho\left(y ; \tau_{y}^{*} \mid \xi_{y}^{*}\right) \leq V^{\prime}(y) \leq \rho\left(y ; \tau \mid \xi_{y}^{*}\right) \leq r(y ; \tau) ; \quad \forall \quad y>0, \tau \in \mathscr{O}_{, T} .
$$

Read (5.22) with $\tau=\tau_{y}^{*}$, and recall (4.18), to obtain (5.1) and part (ii) of Theorem 2.

Under the added assumption (5.18), Lemma 3 (with $\tau=T$ ) and Lemma 5 yield

$$
V^{\prime}(y)=\rho\left(y ; T \mid \xi_{y}^{*}\right) .
$$

Hence, by (5.23), (5.1) and (2.11),

$$
\nu^{*}\left(y \mid \xi_{y}^{*}\right)=\rho\left(y ; T \mid \xi_{y}^{*}\right)-\rho^{*}\left(y \mid \xi_{y}^{*}\right) \equiv 0 .
$$

This proves part (iii) of Theorem 2.

\section{Capital stock dynamics}

Suppose that instead of the simple capital stock equation $Y \equiv y+\xi$ we have, corresponding to any given cumulative investment process $\xi \in \mathscr{\ell}$ and any initial capital stock $y \in[0, \infty)$, the productive capital stock process $\left\{Y_{t}\right\}_{0 \leq t \leq T}$ defined as the unique solution of the stochastic differential equation

$$
\begin{aligned}
& d Y_{t}=-\delta(t) Y_{t} d t+a(t) Y_{t} d W_{t}+d \xi_{t} ; \quad 0 \leq t \leq T, \\
& Y_{0}=y .
\end{aligned}
$$

Here, $W=\left\{W_{t}\right\}_{0 \leq t \leq T}$ is a standard Brownian motion process on $(\Omega, \Im, P$; $\left.\left\{\Im_{t}\right\}\right)$ and $\delta(\cdot)$ and $a(\cdot)$ are bounded by finite constants, uniformly in $(t, \omega)$, and progressively measurable with respect to $\left\{\Im_{t}\right\}$. The process $\delta(\cdot)$ in the drift coefficient may be interpreted as the rate of depreciation, and the proportional 
diffusion coefficient, $a(\cdot)$, as the "standard deviation" per unit time of an exogenous "productivity shock". Extending the results of the previous sections to this more general case is straightforward, but for completeness we provide the details below.

An explicit formula for $Y_{t}$ is given by

$$
Y_{t}=e^{-D(t)} M_{t}\left\{y+\int_{[0, t)} e^{D_{s}} M_{s}^{-1} d \xi_{s}\right\}
$$

where we have used the notation

$$
\begin{gathered}
D_{t} \triangleq \int_{0}^{t} \delta(s) d s \quad ; \quad 0 \leq t \leq T \\
M_{t} \triangleq \exp \left(\int_{0}^{t} a(s) d W_{s}-\frac{1}{2} \int_{0}^{t} a^{2}(s) d s\right) \quad ; \quad 0 \leq t \leq T .
\end{gathered}
$$

The "myopic investor's" solution of (6.1) is given by taking $\xi \equiv 0$ in (6.2), i.e.

$$
X_{t} \triangleq y e^{-D_{t}} M_{t}
$$

Consider the following extension of the definition of the small investor's expected payoff (2.5), where depreciation and productivity changes have been accounted for:

$$
\begin{aligned}
\nu_{d}(y ; \tau \mid \xi) & \triangleq E\left[\int_{\tau}^{T} e^{-D_{t}} M_{t} \pi\left(t, Y_{t}\right) d t+\left(e^{-D_{T}} M_{T} g\left(Y_{T}\right)-e^{-D_{\tau}} M_{\tau} \gamma(\tau)\right) 1_{\{\tau<T\}}\right] ; \\
y & >0, \tau \in \mathscr{O}_{0, T}, \xi \in \mathscr{C},{ }^{4}
\end{aligned}
$$

and the corresponding expected opportunity cost:

$$
\begin{aligned}
\rho_{d}(y ; \tau \mid \xi) & \triangleq E\left[\int_{0}^{\tau} e^{-D_{t}} M_{t} \pi\left(t, Y_{t}\right) d t+e^{-D_{\tau}} M_{\tau} \gamma(\tau) 1_{\{\tau<T\}}+e^{-D_{T}} M_{T} g\left(Y_{T}\right) 1_{\{\tau=T\}}\right] ; \\
y & >0, \tau \in \mathscr{O}_{0, T}, \xi \in \mathscr{C} .
\end{aligned}
$$

Of course (6.6) and (6.7) agree with (2.5) and (2.8), respectively, when $\delta \equiv a \equiv$ 0 , or equivalently, $e^{-D_{t}} \equiv M_{t} \equiv 1$. Note that

$$
\begin{gathered}
\rho_{d}(y ; \tau \mid \xi)=E\left[\int_{0}^{\tau} \tilde{\pi}\left(t, y+\tilde{\xi}_{t}\right) d t+\tilde{\gamma}(\tau) 1_{\{\tau<T\}}+\tilde{g}\left(y+\tilde{\xi}_{T}\right) 1_{\{\tau=T\}}\right] \\
y>0, \tau \in \mathscr{S}_{0, T}, \xi \in \mathscr{C}
\end{gathered}
$$

where, for $y \geq 0, t>0$,

${ }^{4}$ Strictly speaking, the "right" form of the payoff is

$$
\hat{\nu}_{d}(y ; \tau \mid \xi) \triangleq E\left[\int_{\tau}^{T} e^{-D_{\tau, t}} M_{\tau, t} \pi\left(t, Y_{t}\right) d t+\left(e^{-D_{\tau, T}} M_{\tau, T} g\left(Y_{T}\right)-\gamma(\tau)\right) 1_{\{\tau<T\}}\right],
$$

where $D_{s, t}=\int_{s}^{t} \delta(u) d u$ and $M_{S, t}=M_{t} / M_{S}$, but the two formulations are seen to be equivalent by considering the associated dynamic programming equations. 


$$
\begin{aligned}
& \tilde{\pi}(t, y) \triangleq e^{-D_{t}} M_{t} \pi\left(t, e^{-D_{t}} M_{t} \cdot y\right), \\
& \tilde{g}(y) \triangleq e^{-D_{t}} M_{t} g\left(e^{-D_{t}} M_{t} \cdot y\right), \\
& \tilde{\gamma}(t) \triangleq e^{-D_{t}} M_{t} \gamma(t) \\
& \tilde{\xi}_{t} \triangleq \int_{[0, T)} e^{D_{s}} M_{s}^{-1} d \xi_{s} .
\end{aligned}
$$

Define, for $y>0, \tau \in \mathscr{C}_{0, T}, \xi \in \mathscr{C}$,

$$
\begin{aligned}
& \tilde{\nu}(y ; \tau \mid \xi) \triangleq E\left[\int_{\tau}^{T} \tilde{\pi}\left(t, y+\xi_{t}\right) d t-\tilde{\gamma}(\tau) 1_{\{\tau<T\}}+\tilde{g}\left(y+\xi_{T}\right) 1_{\{\tau<T\}}\right], \\
& \tilde{\rho}(y ; \tau \mid \xi) \triangleq E\left[\int_{0}^{T} \tilde{\pi}\left(t, y+\xi_{t}\right) d t+\tilde{\gamma}(\tau) 1_{\{\tau<T\}}+\tilde{g}\left(y+\xi_{T}\right) 1_{\{\tau=T\}}\right] .
\end{aligned}
$$

It is then obvious that

$$
\begin{aligned}
\nu_{d}(y ; \tau \mid \xi) & \equiv \tilde{\nu}(y ; \tau \mid \tilde{\xi}), \\
\rho_{d}(y ; \tau \mid \xi) & \equiv \tilde{\rho}(y ; \tau \mid \tilde{\xi})
\end{aligned}
$$

and since, for $\xi \equiv 0$, we have $\tilde{\xi} \equiv \xi$, we have

$$
\begin{aligned}
& m_{d}(y ; \tau) \triangleq \nu_{d}(y ; \tau \mid 0)=\tilde{\nu}(y ; \tau \mid 0) \triangleq \tilde{m}(y ; \tau), \\
& r_{d}(y ; \tau) \triangleq \rho_{d}(y ; \tau \mid 0)=\tilde{\rho}(y ; \tau \mid 0) \triangleq \tilde{r}(y ; \tau),
\end{aligned}
$$

where the second definition in each line goes from right to left. Therefore, in the myopic investor's case the problem of selecting an investment strategy $\tau \in \mathscr{C}_{0, T}$ to attain the optimum payoff $m_{d}^{*}(y) \triangleq \sup _{\tau \in \mathscr{K}_{0, T}} m(y ; \tau)$, reduces to the myopic investor's problem we have treated in Sects. 2 and 3, where $\delta(\cdot)$ and $a(\cdot)$ were identically zero, but with $\pi, g$, and $\gamma$ replaced by $\tilde{\pi}, \tilde{g}$ and $\tilde{\gamma}$, repectively. Let $\tilde{R}^{y}(t)$ be defined as in (3.1), with $\pi, g$, and $\gamma$ replaced by $\tilde{\pi}, \tilde{g}$ and $\tilde{\gamma}$, repectively. Then we immediately obtain the extension of Proposition 1 to the present, more general, case:

Proposition 2: Assume that (2.1)-(2.4) and (3.2)-(3.3) hold with $\pi, g$, and $\gamma$ replaced by $\tilde{\pi}, \tilde{g}$ and $\tilde{\gamma}$, repectively. Then there exists a submartingale $\tilde{Z}_{0}^{y}(\cdot)$, with sample paths which are almost surely right-continous and admit left-hand limits, and is such that

$$
\tilde{Z}_{0}^{y}(t)=\underset{\tau \in \mathscr{T}_{t, T}}{\operatorname{essinf}} E\left[\tilde{R}^{y}(\tau) \mid \Im_{t}\right], \quad \text { a.s. for all } t \in[0, T] .
$$

Furthermore, $\tilde{Z}_{0}^{y}(t) \leq \tilde{R}^{y}(t)$ a.s. for all $t \in[0, T]$, and the stopping time

$$
\tilde{\sigma}(y) \triangleq \inf \left\{t \in[0, T] ; \tilde{Z}_{0}^{y}(t)=\tilde{R}^{y}(t)\right\}
$$

is an optimal investment strategy for the myopic investor: 


$$
m_{d}(y ; \tilde{\sigma}(y))=m_{d}^{*}(y)
$$

Now consider the social planning problem of Sect. 4 with the capital stock dynamics given by (6.1). The expected social surplus is defined as in (4.1):

$$
J_{d}(y ; \xi) \triangleq E\left[\int_{0}^{T} \Pi\left(t, Y_{t}\right) d t-\int_{[0, T)} \gamma(t) d \xi_{t}+G\left(Y_{T}\right)\right],
$$

where $Y(t), 0 \leq t \leq T$ is now given by (6.2). Define

$$
\begin{aligned}
& \tilde{\Pi}(t, y) \triangleq \Pi\left(t, e^{-D_{t}} M_{t} \cdot y\right) \equiv \int_{1}^{y} \tilde{\pi}(t, x) d x, \\
& \tilde{G}(y) \triangleq G\left(e^{-D_{T}} M_{t} \cdot y\right) \equiv \int_{1}^{y} \tilde{g}(x) d x,
\end{aligned}
$$

and assume (4.4) holds. Then we have,

$$
J_{d}(y ; \xi)=\tilde{J}(y ; \tilde{\xi})
$$

where, for $y>0$ and $\eta \in \mathscr{A}$ :

$$
\tilde{J}(y ; \eta) \triangleq\left[\int_{0}^{T} \tilde{\Pi}\left(t, y+\eta_{t}\right) d t-\int_{[0, T)} \tilde{\gamma}(t) d \eta_{t}+\tilde{G}\left(y+\eta_{T}\right)\right] .
$$

Observe that the mapping $\xi \mapsto \tilde{\xi}: \mathscr{C} \rightarrow \mathscr{C}$ is one-to-one and onto with the inverse given by $\eta \mapsto \int_{[0, t)} e^{-D_{s}} M_{s} d \eta_{s}$. Hence, it is clear that given an optimal process, say $\eta_{y}^{*} \in \mathscr{\ell}$, for the social planner's problem associated with $\tilde{J}(y ; \cdot)$, an application of the inverse of $\xi \mapsto \tilde{\xi}$ to $\eta_{y}^{*}$ will produce an optimal process, say $\xi_{d, y}^{*} \in \mathscr{C}$, to the social planner's problem associated with $J_{d}(y ; \cdot)$. From Proposition 2 and the discussion preceding it it is clear that an optimal process for the former problem is given by

$$
\eta_{y}^{*}(t) \triangleq \sup \{z \in[y, \infty) ; \tilde{\sigma}(z+)<t\}, \quad 0 \leq t \leq T
$$

(cf. (4.15)). Denote the value function of the latter problem by

$$
V_{d}(y) \triangleq \sup _{\xi \in \mathscr{C}} J_{d}(y ; \xi)
$$

Let $I_{d}(y) \triangleq J_{d}(y ; 0)=E\left[\int_{0}^{T} \Pi\left(t, X_{t}\right) d t+G\left(X_{t}\right)\right]$ (cf. (6.5)) and

$$
\xi_{d, y}^{*}(t) \triangleq \int_{[0, t)} e^{-D_{s}} M_{s} d \eta_{s}^{*}, \quad 0 \leq t \leq T .
$$

The following extensions of Theorems 1 and 2 follow easily, now that this notation is in place: 
Theorem 3: Assume that (4.4) holds and that (2.1)-(2.4), (3.2)-(3.3) hold with $\pi$, $g$, and $\gamma$ replaced by $\tilde{\pi}, \tilde{g}$ and $\tilde{\gamma}$, respectively. Then, for all $y>0$, there exists an optimal investment process $\xi_{d, y}^{*}(\cdot)$ for the "social planner's" problem of (6.21), and it is given by (6.22). Furthermore, for all $y>0$,

$$
\begin{gathered}
V_{d}(y)=I_{d}(y)+\int_{y}^{\infty} m_{d}^{*}(x) d x \\
V_{d}^{\prime}(y)=r_{d}^{*}(y) \triangleq \inf _{\tau \in \mathscr{O}_{0, T}} r_{d}(y ; \tau) \\
\tilde{\sigma}(y+)=\inf \left\{t \in[0, T) ; \xi_{d, y}^{*}(t)>0\right\} \wedge T
\end{gathered}
$$

Theorem 4: Assume that (4.4) holds and that (2.1)- (2.4), (3.2)-(3.3) hold with $\pi, g$, and $\gamma$ replaced by $\tilde{\pi}, \tilde{g}$ and $\tilde{\gamma}$, repectively. Then for any given $y>0$ :

(i) We have

$$
V_{d}^{\prime}(y)=r_{d}^{*}(y)=\rho_{d}^{*}\left(y \mid \xi_{d, y}^{*}\right) \triangleq \inf _{\tau \in \mathscr{0}, T} \rho_{d}\left(y ; \tau \mid \xi_{d, y}^{*}\right) .
$$

(ii) The entry time

$$
\tau_{d, y}^{*} \triangleq \inf \left\{t \in[0, T) ; \xi_{d, y}^{*}(t)>0\right\} \wedge T
$$

is optimal both for a "myopic investor" (who forecasts that $\xi \equiv 0$ will prevail) and for a "small investor" who forecasts that the "socially optimal" strategy $\xi \equiv \xi_{d, y}^{*}(\cdot)$ will prevail.

(iii) If, in addition, there exists an $\varepsilon_{0}>0$ such that $\nu_{d}\left(y+\varepsilon_{0} ; T \mid \xi_{d, y}^{*}\right)>-\infty$, then

$$
\nu^{*}\left(y \mid \xi_{d, y}^{*}\right) \triangleq \sup _{\tau \in \mathscr{\mathscr { O }}, T} \nu_{d}\left(y ; \tau \mid \xi_{d, y}^{*}\right)=0 .
$$

\section{Concluding remarks}

The results above can be extended to the case of finite resources, i.e. the case where there is an upper bound on the amount of cumulative investment $\xi$. This extension is straightforward when there is no depreciation of capital stock and its productivity does not change, i.e. in the setting of Sects. 2-5 above. The interested reader is referred to Karatzas (1985) and El Karoui and Karatzas (1988) for the analogous treatment of a related finite-fuel stochastic control problem.

In the setting of Sect. 6 and with finite resources, when capital stock depreciates or is subject to productivity changes, an externality seems to arise unless the resource bound is subject to the same dynamics as the capital stock. The small investor's problem and the social planner's problem are then no longer equivalent, unless the small investor's problem is modified to take this into account. 
Furthermore, myopia is no longer optimal. Rigorous treatment of this problem must await further study.

\section{References}

Arrow, K.J.: Optimal capital policy with irreversible investment. In: Wolfe, J.N. (ed.) Value, capital and growth. Papers in honor of Sir John Hicks. Edinburgh: Edinburgh University Press, 1968, pp 1-19

Baldursson, F.M.: Singular stochastic control and optimal stopping, Stochastics 21, 1-40 (1987)

Baldursson, F.M.: Industry equilibrium and irreversible investment under uncertainty in oligopoly. IOES working paper no. w95:5, Department of Economics, University of Iceland (1995)

Bather, J.A., Chernoff, H.: Sequential decisions in the control of a spaceship. Proc. Fifth Berkeley Symposium on Mathematical Statistics and Probability 3, 181-207 (1966)

Beneš, V.E., Shepp, L.A., Witsenhausen, H.S.: Some solvable stochastic control problems, Stochastics 4, 39-83 (1980)

Bertola, G.: Irreversible investment. Preprint, Princeton University (1989)

Bertola, G., Caballero, R.J.: Irreversibility and aggregate investment. Rev. Econ. Studies 61, 223-246 (1994)

Chow, P.-L., Menaldi, J.-L., Robin, M.: Additive control of stochastic linear systems with finite horizon. SIAM J. Control and Optimization 23 (6), 858-899 (1985)

Dixit, A.: Entry and exit decisions under uncertainty. J. Polit. Econ. 97, 620-638 (1989a)

Dixit, A.: Hysteresis, import penetration and exchange rate pass-through. Q. J. Econ. 26, 309-325 (1989b)

Dixit, A., Pindyck, R.: Investment under uncertainty. Princeton, N.J.: Princeton University Press 1994

El Karoui, N.: Les aspects probabilistes du contrôle stochastique. Lect. Notes Math., vol. 876, pp. 73238. Berlin, Heidelberg, New York: Springer 1981

El Karoui, N., Karatzas, I.: Probabilistic aspects of finite-fuel, reflected follower problems. Acta Appl. Math. 11, 223-258 (1988)

El Karoui, N., Karatzas, I.: A new approach to the Skorohod problem, and its applications. Stochast. Stochast. Rep. 34, 57-82 (1991)

Karatzas, I.: A class of singular stochastic control problems. Adv. Appl. Probab. 15, 225-254 (1983)

Karatzas, I.: Probabilistic aspects of finite fuel stochastic control. Proc. Nat'1. Acad. Sci. USA 82, 5579-5581 (1985)

Karatzas, I.: Probabilistic aspects of optimal stopping and control. Lecture notes, Columbia University (1993)

Karatzas, I., Shreve, S.: Connections between optimal stopping and singular stochastic control, I. Monotone follower problems. SIAM J. Control Optimization 22, 856-877 (1984)

Karatzas, I., Shreve S.: Connections between optimal stopping and singular stochastic control, II. Reflected follower problems, SIAM J. Control Optimization 23, 433-451 (1985)

Karatzas, I., Shreve S.: Equivalent models for finite- fuel stochastic control, Stochastics 18, 245-276 (1986)

Kobila, T.Ø.: Partial investment under uncertainty. In: Lund, D., Øksendal, B. (eds.) Stochastic models and option values. Amsterdam: North-Holland 1991, pp 167-186

Kobila, T.Ø.: A class of solvable stochastic investment problems involving singular controls. Stochastics 43, 29-63 (1993a)

Kobila, T.Ø.: An application of reflected diffusions to the problem of choosing between hydro- and thermal-power generation. Stoch. Processes Appl. 44, 117-139 (1993b)

Leahy, J.: Investment in competitive equilibrium: The optimality of myopic behavior. Q. J. Econ. 30, 1104-1133 (1993)

Lucas, R.E., Prescott, E.C.: Investment under uncertainty. Econometrica 39, 659-681 (1971)

Pindyck, R.: Irreversible investment, capacity choice and the value of the firm. Am. Econ. Rev. 78, 969-985 (1988)

Pindyck, R.: Irreversibility, uncertainty and investment, J. Econ. Literat. 29, 1110-1148 (1991)

Shiryayev, A.N.: Optimal stopping rules. New York: Springer-Verlag 1978

Snell, J.L.: Applications of martingale system theorems, Trans. Am. Math. Soc. 73, 293-312 (1953) 